\title{
A New Mixed Finite Element Method for Biot Consolidation Equations
}

\author{
Luoping Chen ${ }^{1,3}$ and Yan Yang ${ }^{2 *}$ \\ ${ }^{1}$ School of Mathematics, Southwest Jiaotong University, Chengdu, Sichuan 611756, \\ China \\ ${ }^{2}$ School of Sciences, Southwest Petroleum University, Chengdu, Sichuan 610500, China \\ ${ }^{3}$ MOE Key Lab for Neuro Information, School of Life Science and Technology, \\ University of Electronic Science and Technology of China, Chengdu, Sichuan 611731, \\ China
}

Received 15 June 2019; Accepted (in revised version) 1 February 2020

\begin{abstract}
In this paper, we study a new finite element method for poroelasticity problem with homogeneous boundary conditions. The finite element discretization method is based on a three-variable weak form with mixed finite element for the linear elasticity, i.e., the stress tensor, displacement and pressure are unknown variables in the weak form. For the linear elasticity formula, we use a conforming finite element proposed in [11] for the mixed form of the linear elasticity and piecewise continuous finite element for the pressure of the fluid flow. We will show that the newly proposed finite element method maintains optimal convergence order.
\end{abstract}

AMS subject classifications: 65M10, 78A48

Key words: Biot consolidation equations, linear elasticity, finite element method, convergence analysis.

\section{Introduction}

General theory describing the consolidation of a porous elastic soil is very important in application, for example, predicting the behavior of foundation resting on a saturated clay is an important problem in foundation engineering. The foundation allows for the occurrence of finite geometry changes and finite elastic strains during the consolidation process. This theory of poroelasticity addresses the time-dependent coupled process between the deformation of porous materials and the fluid flow inside. The governing equations have been cast in a rate form and laws which determine deformation and pore

*Corresponding author.

Emails: cherrychen@home.swjtu.edu.cn (L. P. Chen), yyan2011@163.com (Y. Yang) 
fluid flow are Hookes's law and Darcy's law. The theoretical basis of consolidation was established by Terzaghi [27], then, Biot generalized the theory to three dimensional transient consolidation $[5,6]$. Since then, poroelastic theory has been used in a diverse range of science and engineering application, for instance, $\mathrm{CO}_{2}$ sequestration in environmental engineering $[15,16]$ are important applications of poroelasticity. Recently, research in poroelasticity has been a surge in activity, not only because of the application described above, but also due to emerging applications in biomechanics engineering such as biological soft tissue modeling including arterial walls, skin, cardiac muscle and articular cartilage $[13,17,25,28]$.

There is an extensive literature on numerical methods for poroelasticity. The most commonly used numerical discretization are based on the two-fields model problem, i.e., displacement $\boldsymbol{u}$ and fluid pressure $p$ as its unknown variables. Standard centered finite difference methods are studied for both one and two dimensional of the model problem $[8,9]$. Also, continuous finite element methods, such as Taylor Hood element and Mini element are employed for the displacement and pressure, see [14,18] as examples. However, it is well known that the approximation by centered difference method and some Galerkin finite element method often exhibit nonphysical oscillation in the pressure of the fluid flow. Therefore, for the two-field model problem, finite difference based on staggered grids [9], cell-centered finite volumn discretization [3,19] are studied and proved to be the stable discretization. For the two-field model problem with classical stable finite element methods (such as Mini, stabilized $P_{1}-P_{1}$ element etc.), the approximate pressure variable still appears nonphysical oscillation under the condition of low permeability or small time step size. Authors in [23] claim that the well known inf-sup stable pair spaces does not necessary provide oscillation-free solution and stabilized term is added to guarantee the monotonicity of the solution to eliminate oscillation. Finite element methods based on classical three-fields model problem, i.e., displacement, fluid flux, pore pressure, are also studied trying to solve the nonphysical oscillations of the fluid pressure. Based on the analysis of locking reason in pure linear elasticity problem, Phillips and Wheeler make arguments that under certain conditions, nonphysical oscillation of fluid pressure may be produced by locking in the solid elasticity. Therefore, nonconforming finite element methods with couple continuous and discontinuous Galerkin (DG) methods for the displacement and a mixed finite element method for the flow variables are investigated, see [20-22]. Stabilized term with face bubble is added in [24] for the finite element (with linear $P_{1}$ element for $\boldsymbol{u}$ and lowest Raviart Thomas element for fluid flux and pressure) to guarantee uniform error bounds. Other three-field based finite element methods are also studied, we refer [26,30] as well as the references therein for further investigation. Four fields formulations for the poroelasticity problem are also investigated. Least squares mixed finite element methods for the stress tensor/displacement/fluid velocity/fluid pressure four-field formulation have been proposed by Korsawe and Starke [12] and Tchonkova et al. [26]. Yi [29] developed a four-field discretization method with displacement, stress tensor in solid subproblem satisfying Hellinger-Reissner variational principle (using Arnold-Winther element) 
and using mixed form for the pressure and flux in flow subproblem (using RaviartThomas element). Theoretical analysis in [29] shows the optimal convergence for the Arnold-Winther and Raviart-Thomas element pairs but numerical experiment shows lose of some convergence order in stress tensor, pressure and flux variables. In recent studies $[4,10]$, the authors also use the Hellinger-Reissner variation for the elasticity of the system and impose weakly the symmetry of the stress tensor resulting in a saddle point problem with stress tensor, displacement, pressure and Lagrange multiplier. The authors prove that the parameter-robust stability of this resulting four-field formulation. The difficulty of the four-field problem is that the solid subproblem does not lend itself trivially to mixed finite element.

Motivated by the finite element exterior calculus theory developed by Douglas Arnold [1,2] and thanks for the distinguished work for linear elasticity by $\mathrm{Hu}[11]$, in this paper, we will investigate the finite element method for the poroelasticity with displacement and stress tensor variables satisfy the subcomplex of the elasticity complex in [2]. We mainly consider the Biot consolidation model problem in two dimensional (2D) spaces with homogeneous boundary condition and solving the Biot consolidation problem with the total stress tensor $\tilde{\sigma}$, the displacement $\boldsymbol{u}$ and the pore pressure $p$ as its unknown variables. We choose the finite element for $\tilde{\sigma}, \boldsymbol{u}$ that by solving the classical Helliger-Reissner mixed problem of the elasticity equations with conforming finite element discretization proposed in [11]. Piecewise continuous finite element method are used for the pore pressure of the model problem. We proved the existence and uniqueness of the solution of this weak form and also make a convergence analysis to the finite discretization of the all the pore pressure, displacement and the tenor stress variables. Theoretical analysis show that the finite element methods maintain optimal convergence order. Several numerical experiments are implemented and verify theoretical analysis.

The outline of the paper is as follows. In Section 2, we will introduce the model problem as well as the weak form and the finite element method. Convergence analysis will be given for the model problem in Section 3. In Section 4, we implement two examples and show the performance of the finite element method numerically.

\section{Model problem and finite element method}

Mechanical processes in poroelasticity mainly contain two basic elements: fluid flow and deformation of elasticity body. Mathematical model for the poroelasticity are derived from physical principles, i.e., the mass conservation and momentum balance for both fluid flow and elasticity body. The momentum balance for the solid phase is given as the following well known equilibrium conditions for the total stress tensor field $\tilde{\sigma}$ :

$$
\operatorname{div} \tilde{\sigma}=-f
$$


where $f$ is the body force per unit volume of the bulk material. From the poroelasticity theory, the effective stress $\sigma$ is defined as

$$
\sigma=\tilde{\sigma}+c_{\text {up }} p \boldsymbol{I}
$$

with $c_{u p}$ being a positive coefficient and assumption of incompressible fluid implies $c_{u p}=$ 1. $p$ is a fluid pressure and $\boldsymbol{I}$ is the identity matrix. Let $\boldsymbol{u}$ denote the displacement variable and $\boldsymbol{\epsilon}(\boldsymbol{u})$ be the strain tensor, the classical strain-displacement relation is defined as

$$
\boldsymbol{\epsilon}(\boldsymbol{u})=\frac{1}{2}\left(\nabla \boldsymbol{u}+(\nabla \boldsymbol{u})^{T}\right) .
$$

One of the constitutive relationship of the model system is presented as follows

$$
\tilde{\sigma}=2 \mu \boldsymbol{\epsilon}(\boldsymbol{u})+\lambda \operatorname{tr}(\boldsymbol{\epsilon}(\boldsymbol{u})) \boldsymbol{I}-c_{u p} p \boldsymbol{I},
$$

with $\lambda, \mu$ being Láme coefficients and tr being the trace operator.

Let $\theta$ denote the increment of fluid volume per unit volume of soil, another constituent equation of the system is presented as:

$$
\theta=c_{p u} \operatorname{div} \boldsymbol{u}+c_{p p} p,
$$

with physical parameter $c_{p u}$ measuring the ratio of fluid volume that squeezed out to the volume of soil change and $c_{p p}$ measures the amount of fluid that can be forced into the soil under pressure while the volume of the soil is kept constant.

The momentum balance of fluid flow satisfies the Darcy law

$$
q=-K \nabla p
$$

with $q$ being specific discharge and $K$ being the permeability coefficient. The mass conservative law of the incompressible fluid is presented as following continuity equation

$$
\frac{\partial \theta}{\partial t}=-\operatorname{div} \boldsymbol{q}+S_{f}
$$

where $S_{f}$ representing the rate of injected volume due to the external fluid source.

From (2.5)-(2.7), we have the following equation

$$
\frac{\partial\left(c_{p u} \operatorname{div} \boldsymbol{u}\right)}{\partial t}+\frac{\partial\left(c_{p p} p\right)}{\partial t}+\operatorname{div}(-K \nabla p)=S_{f}
$$

Since

$$
\begin{array}{ll}
\operatorname{div}(p \cdot \boldsymbol{I})=\operatorname{grad} p, & \operatorname{tr}(\boldsymbol{\epsilon}(\boldsymbol{u}))=\operatorname{div} \boldsymbol{u}, \\
\operatorname{div}[(\operatorname{div} \boldsymbol{u}) \cdot \boldsymbol{I}]=\operatorname{grad} \operatorname{div} \boldsymbol{u}, & \operatorname{div}(\operatorname{grad} \boldsymbol{u})^{T}=\operatorname{grad} \operatorname{div} \boldsymbol{u},
\end{array}
$$


and suppose all the physical parameters $\lambda, \mu, c_{u p}, c_{p u}, c_{p p}, K$ are constants, then, we have

$$
\begin{aligned}
& \operatorname{div} \tilde{\boldsymbol{\sigma}}=-\boldsymbol{f} \Leftrightarrow \operatorname{div}\left(\sigma-c_{u p} p \cdot \boldsymbol{I}\right)=-\boldsymbol{f} \\
\Leftrightarrow & -\operatorname{div}(2 \mu \boldsymbol{\epsilon}+\lambda \operatorname{tr} \boldsymbol{\epsilon} \cdot \boldsymbol{I})+c_{u p} \operatorname{grad} p=f \\
\Leftrightarrow & -2 \mu \operatorname{div} \boldsymbol{\epsilon}-\lambda \operatorname{div}(\operatorname{tr}(\boldsymbol{\epsilon}) \cdot \boldsymbol{I})+c_{u p} \operatorname{grad} p=\boldsymbol{f} \\
\Leftrightarrow & -\mu \operatorname{div} \operatorname{grad} \boldsymbol{u}-\mu \operatorname{div}(\operatorname{grad} \boldsymbol{u})^{T}-\lambda \operatorname{grad} \operatorname{div} \boldsymbol{u}+c_{u p} \operatorname{grad} p=\boldsymbol{f} \\
\Leftrightarrow & -\mu \operatorname{div} \operatorname{grad} \boldsymbol{u}-(\lambda+\mu) \operatorname{grad} \operatorname{div} \boldsymbol{u}+c_{u p} \operatorname{grad} p=\boldsymbol{f} .
\end{aligned}
$$

Together with the mass conservative of fluid, poroelasticity model problem can be also described as following system with displacenent $\boldsymbol{u}$ and pore pressure $p$ as its unknowns,

$$
\left\{\begin{array}{l}
-\mu \operatorname{divgrad} \boldsymbol{u}-(\lambda+\mu) \operatorname{grad} \operatorname{div} \boldsymbol{u}+c_{u p} \operatorname{grad} p=-f \\
\frac{\partial\left(c_{p u} \operatorname{div} \boldsymbol{u}\right)}{\partial t}+\frac{\partial\left(c_{p p} p\right)}{\partial t}+\operatorname{div}(-K \nabla p)=S_{f} .
\end{array}\right.
$$

For simplicity, we concern the following homogeneous boundary condition

$$
p=0, \quad \boldsymbol{u}=0 \quad \text { on } \partial \Omega \times\{t>0\},
$$

and initial condition

$$
p(0)=p^{0}, \quad \boldsymbol{u}(0)=u^{0} \quad \text { in } \Omega .
$$

In this paper, we just study the case with physical parameters $c_{u p}=c_{p u}=c_{p p}=1$ in the model problem.

We first introduce some notations and spaces that used in this study. We denote $(\cdot, \cdot)$ as the inner product in $L^{2}(\Omega)$ and $W^{m, p}$ as standard Sobolev space with norm $\|\cdot\|_{m, p}$ given by

$$
\|v\|_{m, p}^{p}=\sum_{|\alpha| \leq m}\left\|D^{\alpha} v\right\|_{L^{p}(\Omega)}^{p} .
$$

For $p=2$, we let $H^{m}(\Omega)=W^{m, 2}(\Omega),\|\cdot\|_{m}=\|\cdot\|_{m, 2}$ and $\|\cdot\|=\|\cdot\|_{0,2}$. We denote $C$ as a constant that may take different values in different places.

In this paper, we mainly study the Biot consolidation model problem with total stress tensor $\tilde{\sigma}$, displacement $u$ and pore pressure of fluid $p$ as the unknown variables. Notice that

$$
\begin{aligned}
& \tilde{\boldsymbol{\sigma}}=2 \mu \boldsymbol{\epsilon}(\boldsymbol{u})+\lambda \operatorname{tr}(\boldsymbol{\epsilon}(\boldsymbol{u})) \cdot \boldsymbol{I}-p \cdot \boldsymbol{I} \\
& \Rightarrow \frac{1}{2 \mu}\left[\tilde{\boldsymbol{\sigma}}-\frac{\lambda}{2(\lambda+\mu)} \operatorname{tr}(\tilde{\boldsymbol{\sigma}}) \cdot \boldsymbol{I}\right]=\boldsymbol{\epsilon}(\boldsymbol{u})-\frac{1}{2(\lambda+\mu)} p \cdot \boldsymbol{I}, \\
& \operatorname{div} \boldsymbol{u}=\frac{1}{2(\lambda+\mu)} \operatorname{tr}(\tilde{\boldsymbol{\sigma}})+\frac{1}{\lambda+\mu} p \cdot \boldsymbol{I} .
\end{aligned}
$$


Then, the system can be represented as

$$
\begin{aligned}
& \frac{1}{2 \mu}\left[\tilde{\sigma}-\frac{\lambda}{2(\lambda+\mu)} \operatorname{tr}(\tilde{\boldsymbol{\sigma}}) \cdot \boldsymbol{I}\right]-\boldsymbol{\epsilon}(\boldsymbol{u})+\frac{1}{2(\lambda+\mu)} p \cdot \boldsymbol{I}=0, \\
& \operatorname{div} \tilde{\boldsymbol{\sigma}}=-\boldsymbol{f}, \\
& \frac{1}{2(\lambda+\mu)}(\operatorname{tr}(\tilde{\boldsymbol{\sigma}}))_{t}+\left(\frac{1}{\lambda+\mu}+1\right) p_{t}-\operatorname{div}(K \nabla p)=S_{f} .
\end{aligned}
$$

Let

$$
\begin{aligned}
& H(\operatorname{div}, \Omega, \mathbb{S}):=\left\{\left(\begin{array}{ll}
\sigma_{11} & \sigma_{12} \\
\sigma_{21} & \sigma_{22}
\end{array}\right) \in H(\operatorname{div}, \Omega) \mid \sigma_{12}=\sigma_{21}\right\}, \\
& L^{2}\left(\Omega, \mathbb{R}^{2}\right):=\left\{\left(u_{1}, u_{2}\right)^{T} \mid u_{i} \in L^{2}(\Omega), i=1,2\right\}, \\
& H_{0}^{1}(\Omega, \mathbb{R}):=\left\{p \in L^{2}(\Omega)\left|\nabla p \in\left[L^{2}(\Omega)\right]^{2}, p\right|_{\partial \Omega}=0\right\},
\end{aligned}
$$

and suppose the domain $\Omega$ is subdivided by a family of quasi-uniform triangular grids $\mathcal{T}_{h}$ and $T \in \mathcal{T}_{h}$ denotes any element with three edges $\left\{E_{i}\right\}_{i=0}^{2}$. Let $\left\{\boldsymbol{n}_{\boldsymbol{i}}\right\}_{i=0}^{2}$ be the corresponding unit normal vectors of $\left\{E_{i}\right\}_{i=0}^{2}$. Finite element spaces for stress tensor $\tilde{\sigma}$ and displacement $\boldsymbol{u}$ are denotes as $\Sigma_{h}, V_{h}$ which are chosen as in [11] with polynomial order denoting as $k_{1}\left(k_{1} \geq 3\right)$, i.e., the displacement space is the full $C^{-1}-P_{k_{1}-1}$ space

$$
\boldsymbol{V}_{h}=\left\{\boldsymbol{v} \in L^{2}\left(\Omega, \mathbb{R}^{2}\right)|\boldsymbol{v}|_{T} \in P_{k_{1}-1}\left(T, \mathbb{R}^{2}\right), \text { for all } T \in \mathcal{T}_{h}\right\},
$$

where $P_{k_{1}}$ is used as the space of polynomials with degree no more than $k_{1}$. The stress approximate space $\Sigma_{h}$ is the full $C^{0}-P_{k_{1}}$ space enriched by $\left(k_{1}-1\right) H($ div $)$ edge bubble functions on each edge

$$
\begin{gathered}
\Sigma_{h}=\left\{\boldsymbol{\tau} \in H(\operatorname{div}, \Omega, \mathbb{S}), \boldsymbol{\tau}=\boldsymbol{\tau}_{c}+\boldsymbol{\tau}_{b}, \boldsymbol{\tau}_{c} \in H^{1}(\Omega, \mathbf{S}),\right. \\
\left.\left.\boldsymbol{\tau}_{c}\right|_{T} \in P_{k_{1}}(T, S),\left.\boldsymbol{\tau}_{b}\right|_{T} \in \Sigma_{\partial T, b}, \forall T \in \mathcal{T}_{h}\right\},
\end{gathered}
$$

where

$$
\Sigma_{\partial T, b}=\operatorname{span}\left\{\boldsymbol{\tau}_{E_{i}, j}, i=0,1,2, j=1, \cdots, k_{1}-1\right\},
$$

with $\boldsymbol{\tau}_{E_{i}, j}=\phi_{E_{i}, j} \mathbb{T}_{E_{i}},\left(i=0,1,2, j=1, \cdots, k_{1}-1\right)$, and $\phi_{E_{i}, j} \in P_{k_{1}}(T, \mathbb{R})$ being its associated nodal basis function of the Lagrange element of order $k_{1}$ and $\mathbb{T}_{E_{i}}=\boldsymbol{n}_{\boldsymbol{i}}^{\perp} \boldsymbol{n}_{\boldsymbol{i}}^{\perp}$.

The finite element space of pressure $p$ is defined as

$$
\mathbb{P}_{h}=\left\{q \in H_{0}^{1}|q|_{T} \in P_{k_{2}}(T), k_{2} \geq 1\right\},
$$

where $P_{k_{2}}(T)$ representing the space of polynomials with degree less or equal to $k_{2}$.

Since $\forall \tau \in H(\operatorname{div}, \Omega, \mathbb{S})$, the following equations hold,

$$
\begin{aligned}
& (\operatorname{tr}(\tilde{\boldsymbol{\sigma}}) \cdot \boldsymbol{I}, \boldsymbol{\tau})=(\operatorname{tr}(\tilde{\boldsymbol{\sigma}}), \operatorname{tr}(\boldsymbol{\tau})), \quad(p \boldsymbol{I}, \boldsymbol{\tau})=(p, \operatorname{tr}(\boldsymbol{\tau})), \\
& (\boldsymbol{\epsilon}(\boldsymbol{u}), \boldsymbol{\tau})=-(\boldsymbol{u}, \operatorname{div} \boldsymbol{\tau})+(\boldsymbol{u}, \boldsymbol{\tau} \cdot \boldsymbol{n})_{\partial \Omega},
\end{aligned}
$$


then, the weak form of the system is to find $(\tilde{\boldsymbol{\sigma}}(t), \boldsymbol{u}(t), p(t)) \in H(\operatorname{div}, \Omega, \mathbb{S}) \times\left(L^{2}(\Omega)\right)^{2} \times$ $H_{0}^{1}(\Omega)$ for $t>0$ such that the following relation holds

$$
\begin{aligned}
& \left(\frac{1}{2(\lambda+\mu)}(\operatorname{tr}(\tilde{\boldsymbol{\sigma}}))_{t}, q\right)+\left(\left(\frac{1}{\lambda+\mu}+1\right) p_{t}, q\right) \\
& +(K \nabla p, \nabla q)=\left(S_{f}, q\right), \quad \forall q \in H_{0}^{1}(\Omega), \\
& \frac{1}{2 \mu}(\tilde{\boldsymbol{\sigma}}, \boldsymbol{\tau})-\frac{\lambda}{4 \mu(\lambda+\mu)}(\operatorname{tr}(\tilde{\boldsymbol{\sigma}}), \operatorname{tr}(\boldsymbol{\tau}))+(\boldsymbol{u}, \operatorname{div} \boldsymbol{\tau}) \\
& +\left(\frac{1}{2(\lambda+\mu)} p, \operatorname{tr}(\tau)\right)=0, \quad \forall \tau \in H(\operatorname{div}, \Omega, \mathbb{S}), \\
& (\operatorname{div} \tilde{\boldsymbol{\sigma}}, \boldsymbol{v})=-(\boldsymbol{f}, \boldsymbol{v}), \quad \forall \boldsymbol{v} \in\left(L^{2}(\Omega)\right)^{2} \text {. }
\end{aligned}
$$

The semidiscrete weak formula of the system is to find $\left(\tilde{\boldsymbol{\sigma}}_{h}(t), \boldsymbol{u}_{h}(t), p_{h}(t)\right) \in \Sigma_{h} \times \boldsymbol{V}_{h} \times \mathbb{P}_{h}$ with $t>0$ such that the following system holds

$$
\begin{aligned}
& \left(\frac{1}{2(\lambda+\mu)}\left(\operatorname{tr}\left(\tilde{\sigma}_{h}\right)\right)_{t}, q\right)+\left(\left(\frac{1}{\lambda+\mu}+1\right) p_{h, t}, q\right) \\
& +\left(K \nabla p_{h}, \nabla q\right)=\left(S_{f}, q\right), \quad \forall q \in \mathbb{P}_{h}, \\
& \frac{1}{2 \mu}\left(\tilde{\boldsymbol{\sigma}}_{h}, \boldsymbol{\tau}\right)-\frac{\lambda}{4 \mu(\lambda+\mu)}\left(\operatorname{tr}\left(\tilde{\boldsymbol{\sigma}}_{h}\right), \operatorname{tr}(\boldsymbol{\tau})\right)+\left(\boldsymbol{u}_{h}, \operatorname{div} \boldsymbol{\tau}\right) \\
& +\left(\frac{1}{2(\lambda+\mu)} p_{h}, \operatorname{tr}(\tau)\right)=0, \quad \forall \tau \in \Sigma_{h}, \\
& \left(\operatorname{div} \tilde{\sigma}_{h}, \boldsymbol{v}\right)=-(f, \boldsymbol{v}), \quad \forall v \in V_{h} .
\end{aligned}
$$

In the following, we will analyze the existence and uniqueness of the semidiscrete problems.

Let

$$
\tilde{\boldsymbol{\sigma}}_{h}=\sum_{j=1} \tilde{\boldsymbol{\sigma}}_{j}(t) \boldsymbol{\phi}_{\tilde{\boldsymbol{\sigma}}}^{j}(x), \quad \boldsymbol{u}_{h}=\sum_{j=1} \boldsymbol{u}_{j}(t) \boldsymbol{\phi}_{\boldsymbol{u}}^{j}(x), \quad p_{h}=\sum_{j=1} p_{j}(t) \phi_{p}^{j}(x),
$$

then, the system can be written as

$$
\begin{aligned}
& C_{p p} \frac{\partial}{\partial t} \bar{p}+K_{p p} \bar{p}+C_{\tilde{\sigma} p}^{T} \frac{\partial}{\partial t} \overline{\tilde{\sigma}}=\bar{S}_{f}, \\
& C_{\tilde{\sigma} p} \bar{p}+C_{\tilde{\sigma} \tilde{\sigma}} \overline{\tilde{\sigma}}+C_{\tilde{\sigma} u}^{T} \bar{u}=0, \\
& C_{\tilde{\sigma} u} \overline{\tilde{\sigma}}=\bar{f},
\end{aligned}
$$

which can be written as the differential algebraic equations (DAEs) as follows

$$
E \frac{\partial X(t)}{\partial t}+H X(t)=L(t)
$$


where

$$
\begin{array}{ll}
E=\left(\begin{array}{ccc}
C_{p p} & C_{\tilde{\sigma} p}^{T} & 0 \\
0 & 0 & 0 \\
0 & 0 & 0
\end{array}\right), & H=\left(\begin{array}{ccc}
K_{p p} & 0 & 0 \\
C_{\tilde{\sigma} p} & C_{\tilde{\sigma} \tilde{\sigma}} & C_{\tilde{\sigma} u}^{T} \\
0 & C_{\tilde{\sigma} u} & 0
\end{array}\right), \\
X(t)=\left(\begin{array}{c}
\bar{p}(t) \\
\overline{\tilde{\sigma}}(t) \\
\overline{\boldsymbol{u}}(t)
\end{array}\right), & L(t)=\left(\begin{array}{c}
\bar{S}_{f} \\
0 \\
\bar{f}
\end{array}\right) .
\end{array}
$$

From the theory of DAEs [7], if the matrix $k E+H$ is nonsingular for some $k \neq 0$, then, system (2.13) has a solution. For the case when $k=1$, we have

$$
E+H=\left(\begin{array}{ccc}
C_{p p}+K_{p p} & C_{\tilde{\sigma} p}^{T} & 0 \\
C_{\tilde{\sigma} p} & C_{\tilde{\sigma} \tilde{\sigma}} & C_{\tilde{\sigma} u}^{T} \\
0 & C_{\tilde{\sigma} u} & 0
\end{array}\right)=\left(\begin{array}{cc}
A & B^{T} \\
B & 0
\end{array}\right)
$$

From theory of [31], if $\operatorname{ker}(A) \cap \operatorname{ker}(B)=\{0\}$ and $\operatorname{ker}\left(B^{t}\right)=\{0\}, E+H$ is nonsingular. To verify these relations, we first define the following linear functional

$$
\begin{aligned}
& \psi_{A}\left(\left(p_{h}, \tilde{\boldsymbol{\sigma}}_{h}\right),(q, \boldsymbol{\tau})\right) \\
= & \left(\frac{1}{2(\lambda+\mu)} \operatorname{tr}\left(\tilde{\boldsymbol{\sigma}}_{h}\right), q\right)+\left(\left(\frac{1}{\lambda+\mu}+1\right) p_{h}, q\right)+\left(K \nabla p_{h}, \nabla q\right) \\
& \quad+\frac{1}{2 \mu}\left(\tilde{\sigma}_{h}: \tau\right)-\frac{\lambda}{4 \mu(\lambda+\mu)}\left(\operatorname{tr}\left(\tilde{\boldsymbol{\sigma}}_{h}\right), \operatorname{tr}(\boldsymbol{\tau})\right)+\left(\frac{1}{2(\lambda+\mu)} p_{h}, \operatorname{tr}(\boldsymbol{\tau})\right),
\end{aligned}
$$

and

$$
\psi_{B}\left(\left(p_{h}, \tilde{\sigma}_{h}\right), \boldsymbol{v}\right)=\left(\operatorname{div} \tilde{\boldsymbol{\sigma}}_{h}, \boldsymbol{v}\right) .
$$

Then, we have the following results.

Lemma 2.1. For $\psi_{A}$, we have

$$
\psi_{A}\left(\left(p_{h}, \tilde{\sigma}_{h}\right),\left(p_{h}, \tilde{\sigma}_{h}\right)\right) \geq C\left\|p_{h}\right\|_{1}^{2}+\frac{1}{\mu}\left\|\left(\tilde{\sigma}_{h}\right)_{12}\right\|^{2},
$$

where $C$ is determined by permeability coefficient $K$ and

$$
\tilde{\sigma}_{h}=\left(\begin{array}{ll}
\left(\tilde{\sigma}_{h}\right)_{11} & \left(\tilde{\sigma}_{h}\right)_{12} \\
\left(\tilde{\sigma}_{h}\right)_{12} & \left(\tilde{\sigma}_{h}\right)_{22}
\end{array}\right) .
$$

Proof. Let $q=p_{h}, \tau=\tilde{\sigma}_{h}$ in (2.14), then, we have

$$
\begin{aligned}
& \psi_{A}\left(\left(p_{h}, \tilde{\sigma}_{h}\right),\left(p_{h}, \tilde{\sigma}_{h}\right)\right) \\
= & \left(\frac{1}{2(\lambda+\mu)} \operatorname{tr} \tilde{\sigma}_{h}, p_{h}\right)+\left(\left(\frac{1}{\lambda+\mu}+1\right) p_{h}, p_{h}\right)+\left(K \nabla p_{h}, \nabla p_{h}\right) \\
& \quad+\frac{1}{2 \mu}\left(\tilde{\sigma}_{h}, \tilde{\sigma}_{h}\right)-\frac{\lambda}{4 \mu(\lambda+\mu)}\left(\operatorname{tr} \tilde{\sigma}_{h}, \operatorname{tr} \tilde{\sigma}_{h}\right)+\left(\frac{1}{2(\lambda+\mu)} p_{h}, \operatorname{tr} \tilde{\sigma}_{h}\right) .
\end{aligned}
$$


Notice that

$$
\begin{aligned}
& \frac{1}{2 \mu}\left(\tilde{\boldsymbol{\sigma}}_{h}, \tilde{\boldsymbol{\sigma}}_{h}\right)-\frac{\lambda}{4 \mu(\lambda+\mu)}\left(\operatorname{tr} \tilde{\boldsymbol{\sigma}}_{h}, \operatorname{tr} \tilde{\boldsymbol{\sigma}}_{h}\right) \geq \frac{1}{2(\lambda+\mu)}\left(\left\|\left(\tilde{\boldsymbol{\sigma}}_{h}\right)_{11}\right\|^{2}+\left\|\left(\tilde{\boldsymbol{\sigma}}_{h}\right)_{22}\right\|^{2}\right)+\frac{1}{\mu}\left\|\left(\tilde{\boldsymbol{\sigma}}_{h}\right)_{12}\right\|^{2}, \\
& \frac{1}{\lambda+\mu}\left(\operatorname{tr}\left(\tilde{\boldsymbol{\sigma}}_{h}\right), p_{h}\right) \geq-\frac{1}{\lambda+\mu}\left[\frac{1}{2}\left\|p_{h}\right\|^{2}+\frac{1}{2}\left(\left\|\left(\tilde{\sigma}_{h}\right)_{11}\right\|^{2}+\left\|\left(\tilde{\sigma}_{h}\right)_{22}\right\|^{2}\right)\right] .
\end{aligned}
$$

Then, we have the following estimation

$$
\begin{aligned}
\psi_{A}\left(\left(p_{h}, \tilde{\sigma}_{h}\right),\left(p_{h}, \tilde{\sigma}_{h}\right)\right) & \geq\left(\frac{1}{2(\lambda+\mu)}+1\right)\left\|p_{h}\right\|^{2}+\left\|K^{1 / 2} \nabla p_{h}\right\|^{2}+\frac{1}{\mu}\left\|\left(\tilde{\sigma}_{h}\right)_{12}\right\|^{2} \\
& \geq C\left\|p_{h}\right\|_{1}^{2}+\frac{1}{\mu}\left\|\left(\tilde{\sigma}_{h}\right)_{12}\right\| .
\end{aligned}
$$

Thus, we complete the proof.

Then, we have

$$
\operatorname{ker}\left(\psi_{A}\right)=0 \Rightarrow \operatorname{ker}\left(\psi_{A}\right) \cap \operatorname{ker}\left(\psi_{B}\right)=0 .
$$

For $\operatorname{ker}\left(\psi_{B^{T}}\right)$, we need to find $\boldsymbol{v}_{h}$ such that $\forall \boldsymbol{\tau} \in \Sigma_{h},\left(\boldsymbol{v}_{h}, \operatorname{div} \boldsymbol{\tau}\right)=0$. Because of the choice of $\Sigma_{h}, V_{h}$, we have for $\boldsymbol{v}_{h} \in V_{h}$, there exists $\tau^{*} \in \Sigma_{h}$, such that $\operatorname{div} \tau^{*}=v_{h}$, which results in $\left(\boldsymbol{v}_{h}, \operatorname{div} \tau^{*}\right)=\left(\boldsymbol{v}_{h}, \boldsymbol{v}_{h}\right)=0 \Rightarrow \boldsymbol{v}_{h}=0$. Then, we get $\operatorname{ker}\left(\psi_{B^{T}}\right)=0$.

Next, we will discuss the uniqueness of the equation. Suppose $\left(p_{h}^{1}, \tilde{\sigma}_{h}^{1}, \boldsymbol{u}_{h}^{1}\right),\left(p_{h}^{2}, \tilde{\boldsymbol{\sigma}}_{h}^{2}, \boldsymbol{u}_{h}^{2}\right)$ are two solutions satisfying Eqs. (2.12). Let $\left(e_{p}, e_{\tilde{\sigma}}, e_{\boldsymbol{u}}\right)=\left(p_{h}^{1}-p_{h}^{2}, \tilde{\boldsymbol{\sigma}}_{h}^{1}-\tilde{\boldsymbol{\sigma}}_{h}^{2}, \boldsymbol{u}_{h}^{1}-\boldsymbol{u}_{h}^{2}\right)$, then, we have the following system

$$
\begin{aligned}
& \left(\left(\frac{1}{\lambda+\mu}+1\right) \frac{\partial e_{p}}{\partial t}, q\right)+\left(K \nabla e_{p}, \nabla q\right)+\left(\frac{1}{2(\lambda+\mu)} \operatorname{tr} \frac{\partial e_{\tilde{\sigma}}}{\partial t}, q\right)=0 \\
& -\left(\frac{1}{2(\lambda+\mu)} e_{p}, \operatorname{tr} \tau\right)-\left(\mathcal{A} e_{\tilde{\sigma}}, \tau\right)-\left(e_{u}, \operatorname{div} \tau\right)=0 \\
& \left(\operatorname{div} e_{\tilde{\sigma}}, v\right)=0
\end{aligned}
$$

where

$$
\mathcal{A} \tau=\frac{1}{2 \mu}\left[\tau-\frac{\lambda}{2(\lambda+\mu)} \operatorname{tr} \tau I\right] .
$$

First, we have the following results

\section{Lemma 2.2.}

$$
\left\|e_{\tilde{\sigma}}\right\|_{\mathcal{A}}^{2}=:\left(\mathcal{A} e_{\tilde{\sigma}}, e_{\tilde{\sigma}}\right) \leq \frac{1}{\lambda+\mu}\left\|e_{p}\right\|^{2} .
$$

Proof. Let $\tau=e_{\tilde{\sigma}}, v=e_{u}$ in (2.15b), (2.15c) and add the two equations together to get

$$
\begin{gathered}
\quad\left(\frac{1}{2(\lambda+\mu)} e_{p}, \operatorname{tr} e_{\tilde{\sigma}}\right)+\left(\mathcal{A} e_{\tilde{\sigma}}, e_{\tilde{\sigma}}\right)=0 \\
\Rightarrow\left(\mathcal{A} e_{\tilde{\sigma}}, e_{\tilde{\sigma}}\right)=-\frac{1}{2(\lambda+\mu)}\left(e_{p}, \operatorname{tr} e_{\tilde{\sigma}}\right) .
\end{gathered}
$$


Notice $(\mathcal{A} \tau, \tau)=\|\tau\|_{\mathcal{A}}^{2}$, then, from definition of $\mathcal{A} \tau$, it is easy to verify

$$
\frac{1}{2(\lambda+\mu)}\|\boldsymbol{\tau}\|^{2} \leq\|\boldsymbol{\tau}\|_{\mathcal{A}}^{2} \leq \frac{1}{2 \mu}\|\boldsymbol{\tau}\|^{2}
$$

Since $\|\operatorname{tr}(\tau)\|^{2} \leq 2\|\tau\|^{2}$, we have

$$
\begin{aligned}
\left\|e_{\tilde{\sigma}}\right\|_{\mathcal{A}}^{2} & \leq \frac{1}{2(\lambda+\mu)}\left\|e_{p}\right\|^{2}+\frac{1}{8(\lambda+\mu)}\left\|\operatorname{tr}\left(e_{\tilde{\sigma}}\right)\right\|^{2} \\
& \leq \frac{1}{2(\lambda+\mu)}\left\|e_{p}\right\|^{2}+\frac{1}{4(\lambda+\mu)}\left\|e_{\tilde{\sigma}}\right\|^{2} \\
& \leq \frac{1}{2(\lambda+\mu)}\left\|e_{p}\right\|^{2}+\frac{1}{2}\left\|e_{\tilde{\sigma}}\right\|_{\mathcal{A}}^{2}
\end{aligned}
$$

which results in the conclusion in Lemma 2.2.

Now, for the uniqueness of the system, we have the following results

Lemma 2.3. $e_{p}(t), e_{\tilde{\sigma}}(t), e_{u}(t)$ satisfying Eq. (2.15), then,

$$
e_{p}(t)=0, \quad e_{\tilde{\sigma}}(t)=0, \quad e_{\boldsymbol{u}}(t)=0 .
$$

Proof. Let

$$
q=e_{p}, \quad \tau=\frac{\partial e_{\tilde{\sigma}}}{\partial t}, \quad v=e_{u},
$$

in $(2.15 a),(2.15 b),(2.15 c)$ and add them together to get the following results

$$
\left(\frac{1}{\lambda+\mu}+1\right)\left(\frac{\partial}{\partial t} e_{p}, e_{p}\right)+\left\|K \nabla e_{p}\right\|^{2}-\left(\mathcal{A} e_{\tilde{\sigma}}, \frac{\partial}{\partial t} e_{\tilde{\sigma}}\right)=0
$$

Notice that

$$
\begin{aligned}
& \left(\frac{\partial}{\partial t} e_{p}, e_{p}\right)=\frac{\partial}{2 \partial t}\left\|e_{p}\right\|^{2}, \\
& \left(\mathcal{A} e_{\tilde{\sigma}}, \frac{\partial}{\partial t} e_{\tilde{\sigma}}\right)=\frac{\partial}{2 \partial t}\left\|e_{\tilde{\sigma}}\right\|_{\mathcal{A}}^{2},
\end{aligned}
$$

which results in the following relation

$$
\frac{1}{2}\left(\frac{1}{\lambda+\mu}+1\right) \frac{\partial}{\partial t}\left\|e_{p}(t)\right\|^{2}+\left\|K \nabla e_{p}(t)\right\|^{2}=\frac{1}{2} \frac{\partial}{\partial t}\left\|e_{\tilde{\sigma}}\right\|_{\mathcal{A}}^{2},
$$

integrate on both side of the last equation on $(0, t)$ and because of $e_{p}(0)=0, e_{\tilde{\sigma}}(0)=0$, we finally get

$$
\begin{aligned}
& \left(\frac{1}{2(\lambda+\mu)}+\frac{1}{2}\right)\left\|e_{p}(t)\right\|^{2}+\int_{0}^{t}\|K \nabla p\|^{2} d s=\frac{1}{2}\left\|e_{\tilde{\sigma}}(t)\right\|_{\mathcal{A}}^{2} \\
\Leftrightarrow & \frac{1}{2}\left\|e_{p}(t)\right\|^{2}+\int_{0}^{t}\left\|K \nabla e_{p}(t)\right\|^{2} d s \leq 0 \\
\Leftrightarrow & e_{p}(t)=0,
\end{aligned}
$$


then, from Lemma 2.2, we get

$$
e_{\tilde{\sigma}}(t)=0
$$

Then, from $(2.15 b)$, we have

$$
\left(e_{\boldsymbol{u}}(t), \operatorname{div} \boldsymbol{\tau}\right)=0, \quad \forall \boldsymbol{\tau} \in \Sigma_{h} .
$$

Fixed $t$, because of the choice of $\Sigma_{h}, V_{h}$, there exists $\bar{\tau}$ such that div $\bar{\tau}=e_{u}$, which results in $e_{u}(t)=0$ and we get the results in Lemma 2.3.

For the existence and uniqueness of the fully discretization formula

$$
\begin{array}{ll}
\left(\frac{1}{2(\lambda+\mu)} \operatorname{tr}(\tilde{\boldsymbol{\sigma}})_{h}^{n}, q\right)+\left(\left(\frac{1}{\lambda+\mu}+1\right) p_{h}^{n}, q\right)+\Delta t\left(K \nabla p_{h}^{n}, \nabla q\right) & \\
=\left(\frac{1}{2(\lambda+\mu)} \operatorname{tr}(\tilde{\boldsymbol{\sigma}})_{h}^{n-1}, q\right)+\left(\left(\frac{1}{\lambda+\mu}+1\right) p_{h}^{n-1}, q\right)+\left(S_{f}, q\right), & \forall q \in \mathbb{P}_{h}, \\
\frac{1}{2 \mu}\left(\tilde{\boldsymbol{\sigma}}_{h}^{n}, \boldsymbol{\tau}\right)-\frac{\lambda}{4 \mu(\lambda+\mu)}\left(\operatorname{tr}\left(\tilde{\boldsymbol{\sigma}}_{h}^{n}\right), \operatorname{tr}(\boldsymbol{\tau})\right)+\left(\boldsymbol{u}_{h}^{n}, \operatorname{div} \boldsymbol{\tau}\right) & \\
\quad+\left(\frac{1}{2(\lambda+\mu)} p_{h}^{n}, \operatorname{tr}(\boldsymbol{\tau})\right)=0, & \forall \boldsymbol{\tau} \in \Sigma_{h}, \\
\left(\operatorname{div} \tilde{\boldsymbol{\sigma}}_{h}^{n}, \boldsymbol{v}\right)=-(f, \boldsymbol{v}), & \forall \boldsymbol{v} \in \boldsymbol{V}_{h},
\end{array}
$$

we have the matrix form as follows

$$
C X^{n}=L^{n}(t),
$$

where

$$
\begin{aligned}
C & =\left(\begin{array}{ccc}
C_{p p}+\Delta t K_{p p} & C_{\tilde{\sigma} p}^{T} & 0 \\
C_{\tilde{\sigma} p} & C_{\tilde{\sigma} \tilde{\sigma}} & C_{\tilde{\sigma} u}^{T} \\
0 & C_{\tilde{\sigma} u} & 0
\end{array}\right), \\
X^{n} & =\left(\begin{array}{c}
\bar{p}_{h}^{n} \\
\bar{\sigma}_{h}^{n} \\
\overline{\boldsymbol{u}}_{h}^{n}
\end{array}\right), \quad L^{n}(t)=\left(\begin{array}{c}
\bar{S}_{f}^{n}+C_{p p} p_{h}^{n-1}+C_{\tilde{\sigma} p} \tilde{\sigma}^{n-1} \\
0 \\
\bar{f}^{n}
\end{array}\right) .
\end{aligned}
$$

At each time step, the existence and uniqueness of the system is equivalent to the invertibility of the matrix $C$ which can be proved in the similar way as for the semidiscrete case.

\section{Convergence analysis}

In this section, we will make a convergence analysis of the finite element methods for Biot consolidation problems. In order to make a theoretical analysis of the finite element 
method, we first define the projection operators $R_{h}^{p}: H_{0}^{1}(\Omega, \mathbb{R}) \rightarrow \mathbb{P}_{h}, R_{h}^{\tilde{\sigma}}: H(\operatorname{div}, \Omega, \mathbb{S}) \rightarrow \Sigma_{h}$, $R_{h}^{u}: L^{2}\left(\Omega, \mathbb{R}^{2}\right) \rightarrow V_{h}$ for any $t>0$ such that the following equations hold

$$
\begin{array}{ll}
\left(K \nabla R_{h}^{p} p, \nabla q\right)=\left(S_{f}-\left(\frac{1}{\lambda+\mu}+1\right) p_{t}-\frac{1}{2(\lambda+\mu)} \operatorname{tr} \tilde{\sigma}_{t}, q\right), & \forall q \in \mathbb{P}_{h}, \\
\left(\frac{1}{2(\lambda+\mu)} R_{h}^{p} p, \operatorname{tr} \boldsymbol{\tau}\right)+\left(\mathcal{A} R_{h}^{\tilde{\sigma}} \tilde{\boldsymbol{\sigma}}, \boldsymbol{\tau}\right)+\left(R_{h}^{u} u, \operatorname{div} \boldsymbol{\tau}\right)=0, & \forall \boldsymbol{\tau} \in \Sigma_{h}, \\
\left(\operatorname{div}\left(R_{h}^{\tilde{\sigma}} \tilde{\boldsymbol{\sigma}}\right), \boldsymbol{v}\right)=(f, \boldsymbol{v}), & \forall \boldsymbol{v} \in \boldsymbol{V}_{h},
\end{array}
$$

then, we have

$$
\begin{array}{ll}
\left(K \nabla\left(p-R_{h}^{p} p\right), \nabla q\right)=0, & \forall q \in \mathbb{P}_{h}, \\
\left(\frac{1}{2(\lambda+\mu)}\left(p-R_{h}^{p} p\right), \operatorname{tr} \boldsymbol{\tau}\right)+\left(\mathcal{A}\left(\tilde{\boldsymbol{\sigma}}-R_{h}^{\tilde{\sigma}} \tilde{\boldsymbol{\sigma}}\right), \boldsymbol{\tau}\right)+\left(\boldsymbol{u}-R_{h}^{u} \boldsymbol{u}, \operatorname{div} \boldsymbol{\tau}\right)=0, & \forall \boldsymbol{\tau} \in \Sigma_{h}, \\
\left(\operatorname{div}\left(\tilde{\boldsymbol{\sigma}}-R_{h}^{\tilde{\sigma}} \tilde{\boldsymbol{\sigma}}\right), \boldsymbol{v}\right)=0, & \forall \boldsymbol{v} \in \boldsymbol{V}_{h} .
\end{array}
$$

From (3.1a) and (3.1c), we can easily have the following results for the projection

$$
\begin{aligned}
& \left\|p-R_{h}^{p} p\right\| \leq C h^{k_{2}+1}\|p\|_{k_{2}+1}, \\
& \left\|\tilde{\boldsymbol{\sigma}}-R_{h}^{\tilde{\sigma}} \tilde{\boldsymbol{\sigma}}\right\| \leq C h^{k_{1}+1}\left(\|\tilde{\boldsymbol{\sigma}}\|_{k_{1}+1}+\|\nabla \cdot \tilde{\boldsymbol{\sigma}}\|_{k_{1}+1}\right) .
\end{aligned}
$$

In order to get the error estimation of $\boldsymbol{u}-R_{h}^{u} \boldsymbol{u}$, we need to define the $L^{2}$ projection of $\boldsymbol{u}$ as follows

$$
\left(\boldsymbol{u}-Q_{h} \boldsymbol{u}, \boldsymbol{v}\right)=0, \quad \forall \boldsymbol{v} \in \boldsymbol{V}_{h} .
$$

Then, since $\operatorname{div} \tau \in V_{h}$, from (3.1b), we have

$$
\left(\frac{1}{2(\lambda+\mu)}\left(p-R_{h}^{p} p\right), \operatorname{tr} \boldsymbol{\tau}\right)+\left(\mathcal{A}\left(\tilde{\boldsymbol{\sigma}}-R_{h}^{\tilde{\sigma}} \tilde{\boldsymbol{\sigma}}\right), \boldsymbol{\tau}\right)+\left(Q_{h} \boldsymbol{u}-R_{h}^{u} \boldsymbol{u}, \operatorname{div} \boldsymbol{\tau}\right)=0, \quad \forall \boldsymbol{\tau} \in \Sigma_{h} .
$$

There exists $\boldsymbol{\tau}^{*} \in \Sigma_{h}$, such that $\operatorname{div} \boldsymbol{\tau}^{*}=Q_{h} \boldsymbol{u}-R_{h}^{u} \boldsymbol{u}$ and $\left\|\boldsymbol{\tau}^{*}\right\|_{1} \leq\left\|Q_{h} \boldsymbol{u}-R_{h}^{u} \boldsymbol{u}\right\|$. Then, we have the following results

$$
\begin{aligned}
& \left\|Q_{h} \boldsymbol{u}-R_{h}^{u} \boldsymbol{u}\right\|^{2}=\left(Q_{h} \boldsymbol{u}-R_{h}^{u} u, \operatorname{div} \boldsymbol{\tau}^{*}\right) \\
= & -\left(\frac{1}{2(\lambda+\mu)}\left(p-R_{h}^{p} p\right), \operatorname{tr} \tau^{*}\right)-\left(\mathcal{A}\left(\tilde{\boldsymbol{\sigma}}-R_{h}^{\tilde{\sigma}} \tilde{\boldsymbol{\sigma}}\right), \boldsymbol{\tau}^{*}\right) \\
\leq & \left.C\left(\left\|p-R_{h}^{p} p\right\|+\| \tilde{\sigma}-R_{h}^{\tilde{\sigma}} \tilde{\sigma}\right) \|\right)\left\|\boldsymbol{\tau}^{*}\right\|_{1} \\
\leq & \left.C\left(\left\|p-R_{h}^{p} p\right\|+\| \tilde{\sigma}-R_{h}^{\tilde{\sigma}} \tilde{\sigma}\right) \|\right)\left\|Q_{h} \boldsymbol{u}-R_{h}^{u} \boldsymbol{u}\right\|,
\end{aligned}
$$

which results in

$$
\left.\left\|Q_{h} \boldsymbol{u}-R_{h}^{u} \boldsymbol{u}\right\| \leq C\left(\left\|p-R_{h}^{p} p\right\|+\| \tilde{\boldsymbol{\sigma}}-R_{h}^{\tilde{\sigma}} \tilde{\boldsymbol{\sigma}}\right) \|\right) .
$$


Therefore, we have

$$
\left\|\boldsymbol{u}-R_{h}^{u} \boldsymbol{u}\right\| \leq\left\|\boldsymbol{u}-Q_{h} \boldsymbol{u}\right\|+\left\|Q_{h} \boldsymbol{u}-R_{h}^{u} \boldsymbol{u}\right\| \leq C\left(h^{k_{1}+1}+h^{k_{2}+1}\right) .
$$

It is also easy to get the following results

$$
\begin{aligned}
& \left\|p_{t}-R_{h}^{p} p_{t}\right\| \leq C h^{k_{2}+1}, \\
& \left\|\tilde{\boldsymbol{\sigma}}_{t}-R_{h}^{\tilde{\sigma}} \tilde{\boldsymbol{\sigma}}_{t}\right\| \leq C h^{k_{1}+1}, \\
& \left\|\boldsymbol{u}_{t}-R_{h}^{u} \boldsymbol{u}_{t}\right\| \leq C\left(h^{k_{1}+1}+h^{k_{2}+1}\right) .
\end{aligned}
$$

\subsection{Convergence analysis of semidiscrete problem}

We have the following results for the semidiscrete solution.

Theorem 3.1. Let $p_{h}, \tilde{\boldsymbol{\sigma}}_{h}, \boldsymbol{u}_{h}$ be the semidiscrete numerical solutions satisfying (2.12) and $p, \tilde{\boldsymbol{\sigma}}$, $\boldsymbol{u}$ be the exact solutions, then, we have the following results

$$
\left\|p-p_{h}\right\|^{2}+\int_{0}^{t}\left\|K^{1 / 2} \nabla\left(p-p_{h}\right)\right\|^{2} d s \leq C\left(h^{2\left(k_{1}+1\right)}+h^{2\left(k_{2}+1\right)}\right),
$$

with $C$ depends on Lame constants $\lambda, \mu$ and the regularity of the solutions $p, \tilde{\sigma}$ of the problem but independent of discretization parameters.

Proof. We have the following error equations

$$
\begin{aligned}
& \left(\left(\frac{1}{\lambda+\mu}+1\right)\left(p_{t}-p_{h t}\right), q\right)+\left(K \nabla\left(p-p_{h}\right), \nabla q\right)+\left(\frac{1}{2(\lambda+\mu)}\left(\operatorname{tr} \tilde{\boldsymbol{\sigma}}_{t}-\operatorname{tr} \tilde{\boldsymbol{\sigma}}_{h t}\right), q\right)=0 \\
& -\left(\frac{1}{2(\lambda+\mu)}\left(p-p_{h}\right), \operatorname{tr} \boldsymbol{\tau}\right)-\left(\mathcal{A}\left(\tilde{\boldsymbol{\sigma}}-\tilde{\boldsymbol{\sigma}}_{h}\right), \boldsymbol{\tau}\right)-\left(\boldsymbol{u}-\boldsymbol{u}_{h}, \operatorname{div} \boldsymbol{\tau}\right)=0 \\
& \left(\operatorname{div}\left(\tilde{\boldsymbol{\sigma}}-\tilde{\boldsymbol{\sigma}}_{h}\right), \boldsymbol{v}\right)=0
\end{aligned}
$$

By using the definition of the projection $R_{h}^{p}, R_{h}^{\tilde{\sigma}}, R_{h}^{u}$, denote $\xi_{p}(t)=R_{h}^{p} p-p_{h}, \xi_{\tilde{\sigma}}(t)=$ $R_{h}^{\tilde{\sigma}} \tilde{\sigma}-\tilde{\sigma}_{h}, \xi_{\boldsymbol{u}}(t)=R_{h}^{u} \boldsymbol{u}-\boldsymbol{u}_{h}$ with $t>0$, then, we have the following relation

$$
\begin{aligned}
& \left(\left(\frac{1}{\lambda+\mu}+1\right) \frac{\partial\left(p-R_{h}^{p} p+\xi_{p}\right)}{\partial t}, q\right)+\left(K \nabla \xi_{p}, \nabla q\right) \\
& +\left(\frac{1}{2(\lambda+\mu)} \operatorname{tr} \frac{\partial\left(\tilde{\sigma}-R_{h}^{\tilde{\sigma}} \tilde{\sigma}+\xi_{\tilde{\sigma}}\right)}{\partial t}, q\right)=0 \\
& -\left(\frac{1}{2(\lambda+\mu)} \xi_{p}, \operatorname{tr} \boldsymbol{\tau}\right)-\left(\mathcal{A} \xi_{\tilde{\sigma}}, \boldsymbol{\tau}\right)-\left(\xi_{u}, \operatorname{div} \boldsymbol{\tau}\right)=0 \\
& \left(\operatorname{div} \xi_{\tilde{\sigma}, \boldsymbol{v})}=0 .\right.
\end{aligned}
$$


Choose $q=\xi_{p}, \tau=\xi_{\tilde{\sigma} t}, v=\xi_{u}$ in the equations and add the equations together to get the following results

$$
\begin{aligned}
& \left(\frac{1}{2(\lambda+\mu)}+\frac{1}{2}\right) \frac{\partial}{\partial t}\left\|\xi_{p}\right\|^{2}+\left\|K^{1 / 2} \nabla \xi_{p}\right\|^{2} \\
=- & \left(\frac{1}{2(\lambda+\mu)}\left(\tilde{\sigma}_{t}-R_{h}^{\tilde{\sigma}} \tilde{\sigma}_{t}\right), \xi_{p}\right)+\left(\mathcal{A} \xi_{\tilde{\sigma}},\left(\tilde{\zeta}_{\tilde{\sigma}}\right)_{t}\right) \\
& -\left(\left(\frac{1}{\lambda+\mu}+1\right)\left(p_{t}-R_{h}^{p} p_{t}\right), \xi_{p}\right) .
\end{aligned}
$$

Notice

$$
\left(\mathcal{A} \xi_{\tilde{\sigma}},\left(\xi_{\tilde{\sigma}}\right)_{t}\right)=\frac{1}{2} \frac{\partial}{\partial t}\left\|\xi_{\tilde{\sigma}}\right\|_{\mathcal{A}}^{2}
$$

and by choosing $\tau=\xi_{\tilde{\sigma}}, v=\xi_{u}$ in (3.3b), we can get the following estimation

$$
\left\|\xi_{\tilde{\sigma}}\right\|_{\mathcal{A}}^{2} \leq \frac{1}{\lambda+\mu}\left\|\xi_{p}\right\|^{2}
$$

Then, integrate on $(0, t)$ on both side of the equation, and since $\xi_{p}(0)=0, \xi_{\tilde{\sigma}}(0)=0$ and also the property of the projection, by using the Young's inequality, we finally get the following estimation

$$
\begin{aligned}
& \left\|\xi_{p}(t)\right\|^{2}+\int_{0}^{t}\left\|K^{1 / 2} \nabla \xi_{p}\right\|^{2} d s \\
\leq & C\left(h^{2\left(k_{1}+1\right)}+h^{2\left(k_{2}+1\right)}\right)\left(\left\|\tilde{\sigma}_{t}\right\|_{k_{1}+1}^{2}+\left\|\nabla \cdot \tilde{\sigma}_{t}\right\|_{k_{1}+1}^{2}+\left\|p_{t}\right\|_{k_{2}+1}^{2}\right) .
\end{aligned}
$$

Together with property of projection $R_{h}^{p}$, we finally get the results.

From (3.4) and the relation

$$
\frac{1}{2(\lambda+\mu)}\|\tau\|^{2} \leq\|\tau\|_{\mathcal{A}}^{2} \leq \frac{1}{2 \mu}\|\tau\|^{2}
$$

we can also derive the error estimation of $\left\|\tilde{\sigma}-\tilde{\sigma}_{h}\right\|_{\mathcal{A}}$, i.e.,

$$
\left\|\tilde{\sigma}-\tilde{\sigma}_{h}\right\|_{\mathcal{A}} \leq\left\|\tilde{\sigma}-R_{h}^{\tilde{\sigma}} \tilde{\sigma}\right\|_{\mathcal{A}}+\frac{1}{\lambda+\mu}\left\|\xi_{p}\right\| \leq C\left(h^{k_{1}+1}+h^{k_{2}+1}\right),
$$

where $C$ depends on $\lambda, \mu$ and regularity of exact solutions but independent of $h$.

For the estimation of displacement $u$, we first need to introduce the projection operator $\Pi_{h}: H(\operatorname{div}, \Omega, \mathrm{S}) \rightarrow \Sigma_{h}$ that defined as follows

$$
\left(\operatorname{div}\left(\boldsymbol{\tau}-\Pi_{h} \boldsymbol{\tau}\right), \boldsymbol{v}\right)=0, \quad \forall \boldsymbol{v} \in \boldsymbol{V}_{h},
$$


and the stability

$$
\left\|\Pi_{h} \boldsymbol{\tau}\right\|_{1} \leq\|\boldsymbol{\tau}\|_{1} .
$$

We first estimate $\xi_{\boldsymbol{u}}=R_{h}^{u} \boldsymbol{u}-\boldsymbol{u}_{h}$. Recall that there exist $\boldsymbol{\tau}^{*} \in H(\operatorname{div}, \Omega, \mathrm{S})$ such that $-\operatorname{div} \boldsymbol{\tau}^{*}=$ $\xi_{\boldsymbol{u}}$ and $\left\|\tau^{*}\right\|_{1} \leq C\left\|\xi_{\boldsymbol{u}}\right\|$, then, from (3.3b), we have

$$
\begin{aligned}
& \left\|\xi_{\boldsymbol{u}}\right\|^{2}=\left(-\xi_{\boldsymbol{u}}, \operatorname{div} \boldsymbol{\tau}^{*}\right)=-\left(\xi_{\boldsymbol{u}}, \operatorname{div} \Pi_{h} \boldsymbol{\tau}^{*}\right) \\
= & \left(\mathcal{A}\left(\tilde{\boldsymbol{\sigma}}-\tilde{\boldsymbol{\sigma}}_{h}\right), \Pi_{h} \boldsymbol{\tau}^{*}\right)+\left(\frac{1}{2(\lambda+\mu)}\left(p-p_{h}\right), \operatorname{tr}\left(\Pi_{h} \boldsymbol{\tau}^{*}\right)\right) \\
\leq & C\left(\left\|\tilde{\boldsymbol{\sigma}}-\tilde{\sigma}_{h}\right\|+\left\|p-p_{h}\right\|\right)\left\|\Pi_{h} \tau^{*}\right\| \\
\leq & C\left(\left\|\tilde{\boldsymbol{\sigma}}-\tilde{\sigma}_{h}\right\|+\left\|p-p_{h}\right\|\right)\left\|\xi_{\boldsymbol{u}}\right\|,
\end{aligned}
$$

then, we have

$$
\left\|\boldsymbol{u}-\boldsymbol{u}_{h}\right\| \leq C\left(h^{k_{1}+1}+h^{k_{2}+1}\right) .
$$

\subsection{Convergence analysis of fully discrete problems}

In this subsection, we will make a convergence analysis of the fully discrete formula of the Biot consolidation problems. We have the following main results.

Theorem 3.2. Let $p^{n}, \tilde{\sigma}^{n}, \boldsymbol{u}^{n}$ be the solution satisfying Eq. (2.11) at $t=t_{n}$ and $p_{h}^{n}, \tilde{\sigma}_{h}^{n}, \boldsymbol{u}_{h}^{n}$ are the fully discrete solution satisfying Eq. (2.16), if $\lambda+\mu>1$, then, for $m \geq 1$, we have

$$
\left\|p^{m}-p_{h}^{m}\right\|^{2}+C \sum_{n=1}^{m} \Delta t\left\|K^{1 / 2} \nabla p^{n}\right\|^{2} \lesssim(\Delta t)^{2}+h^{2\left(k_{1}+1\right)}+h^{2\left(k_{2}+1\right)} .
$$

Proof. Subtract Eqs. (2.16) from (2.11) at $t=t_{n}$ to get the following relation

$$
\begin{aligned}
& \left(\frac{1}{2(\lambda+\mu)} \operatorname{tr}\left(\tilde{\boldsymbol{\sigma}}_{t}^{n}-\partial_{t} \tilde{\boldsymbol{\sigma}}_{h}^{n}\right), q\right)+\left(\left(\frac{1}{\lambda+\mu}+1\right)\left(p_{t}^{n}-\partial_{t} p_{h}^{n}\right), q\right)+\left(K \nabla\left(p^{n}-p_{h}^{n}\right), \nabla q\right)=0, \\
& \left(\frac{1}{2(\lambda+\mu)}\left(p^{n}-p_{h}^{n}\right), \operatorname{tr} \boldsymbol{\tau}\right)+\left(\mathcal{A}\left(\tilde{\boldsymbol{\sigma}}^{n}-\tilde{\boldsymbol{\sigma}}_{h}^{n}\right), \boldsymbol{\tau}\right)+\left(\boldsymbol{u}^{n}-\boldsymbol{u}_{h}^{n}, \operatorname{div} \boldsymbol{\tau}\right)=0, \\
& \left(\operatorname{div}\left(\tilde{\boldsymbol{\sigma}}-\tilde{\boldsymbol{\sigma}}_{h}^{n}\right), \boldsymbol{v}\right)=0 .
\end{aligned}
$$

By using the definition of projectors $R_{h}^{p}, R_{h}^{\tilde{\sigma}}, R_{h}^{u}$, and denote $\xi_{p}^{n}=R_{h}^{p} p^{n}-p_{h}^{n}, \xi_{\tilde{\sigma}}^{n}=R_{h}^{\tilde{\sigma}} \tilde{\sigma}^{n}-\tilde{\sigma}_{h}^{n}$, $\xi_{\boldsymbol{u}}^{n}=R_{h}^{u} \boldsymbol{u}^{n}-\boldsymbol{u}_{h}^{n}$, then, we have the following results

$$
\begin{aligned}
& \left(\frac{1}{2(\lambda+\mu)} \partial_{t} \operatorname{tr}\left(\xi_{\tilde{\sigma}}^{n}\right), q\right)+\left(\left(\frac{1}{\lambda+\mu}+1\right) \partial_{t} \xi_{p}^{n}, q\right)+\left(K \nabla \xi_{p}^{n}, \nabla q\right) \\
& =\left(\operatorname{tr}\left(\partial_{t} \tilde{\sigma}^{n}-\tilde{\sigma}_{t}^{n}-\partial_{t}\left(\tilde{\sigma}^{n}-R_{h}^{\tilde{\sigma}} \tilde{\sigma}^{n}\right)\right), q\right)+\left(\partial_{t} p^{n}-p_{t}^{n}-\partial_{t}\left(p^{n}-R_{h}^{p} p^{n}\right), q\right), \\
& -\left(\frac{1}{2(\lambda+\mu)} \xi_{p}^{n}, \operatorname{tr} \boldsymbol{\tau}\right)-\left(\mathcal{A} \xi_{\tilde{\sigma}}^{n}, \boldsymbol{\tau}\right)-\left(\xi_{u}^{n}, \operatorname{div} \boldsymbol{\tau}\right)=0, \\
& \left(\operatorname{div} \xi_{\tilde{\sigma}, \boldsymbol{v}}^{n}\right)=0 .
\end{aligned}
$$


First, by choosing $\tau=\xi_{\tilde{\sigma}}^{n}, v=\xi_{u}^{n}$ in (3.6b) and (3.6c), it is easy to verify the following result

$$
\left\|\xi_{\tilde{\sigma}}^{n}\right\|_{\mathcal{A}} \leq \frac{1}{\lambda+\mu}\left\|\xi_{p}^{n}\right\|^{2}
$$

Notice that (3.6c) also holds at $t=t_{n-1}$, then, choose $\tau=\xi_{\tilde{\sigma}}^{n-1}$ in (3.6b) and $v=\xi_{u}^{n}$, we have the following relation

$$
\left(\mathcal{A} \xi_{\tilde{\sigma}}^{n}, \operatorname{tr}\left(\xi_{\tilde{\sigma}}^{n-1}\right)\right)=-\frac{1}{2(\lambda+\mu)}\left(\xi_{p}^{n}, \operatorname{tr}\left(\xi_{\tilde{\sigma}}^{n-1}\right)\right) .
$$

By Young's inequality and (3.7), we have the following estimation

$$
\begin{aligned}
& \left(\xi_{p}^{n}, \operatorname{tr}\left(\xi_{\tilde{\sigma}}^{n-1}\right)\right) \leq \frac{\epsilon}{2}\left\|\xi_{p}^{n}\right\|^{2}+\frac{1}{2 \epsilon}\left\|\operatorname{tr}\left(\xi_{\tilde{\sigma}}^{n-1}\right)\right\|^{2} \\
\leq & \frac{\epsilon}{2}\left\|\xi_{p}^{n}\right\|^{2}+\frac{\lambda+\mu}{\epsilon}\left\|\xi_{\tilde{\sigma}}^{n-1}\right\|_{\mathcal{A}}^{2} \leq \frac{\epsilon}{2}\left\|\xi_{p}^{n}\right\|^{2}+\frac{1}{\epsilon}\left\|\xi_{p}^{n-1}\right\|^{2},
\end{aligned}
$$

then, we have the following estimation

$$
\left(\mathcal{A} \xi_{\tilde{\sigma}}^{n}, \xi_{\tilde{\sigma}}^{n-1}\right) \geq-\frac{\epsilon}{4(\lambda+\mu)}\left\|\xi_{p}^{n}\right\|^{2}-\frac{1}{\epsilon(\lambda+\mu)}\left\|\xi_{p}^{n-1}\right\|^{2} .
$$

Now, choose $q=\xi_{p}^{n}, \tau=\partial_{t} \xi_{\tilde{\sigma}}^{n}, v=\xi_{u}^{n}$ in (3.6a), (3.6b), (3.6c), respectively and add the three equations together to get the following relation

$$
\begin{aligned}
& \left(\frac{1}{\lambda+\mu}+1\right)\left(\partial_{t} \xi_{p}^{n}, \xi_{p}^{n}\right)+\left(K \nabla \xi_{p}^{n}, \nabla \xi_{p}^{n}\right)-\left(\mathcal{A} \xi_{\tilde{\sigma}}^{n}, \partial_{t} \xi_{\tilde{\sigma}}^{n}\right) \\
= & \left(\operatorname{tr}\left(\partial_{t} \tilde{\sigma}^{n}-\tilde{\sigma}_{t}^{n}-\partial_{t}\left(\tilde{\sigma}^{n}-R_{h}^{\tilde{\sigma}} \tilde{\sigma}^{n}\right)\right), \xi_{p}^{n}\right)+\left(\partial_{t} p^{n}-p_{t}^{n}-\partial_{t}\left(p^{n}-R_{h}^{p} p^{n}\right), \xi_{p}^{n}\right) .
\end{aligned}
$$

Notice that

$$
\left(\frac{1}{\lambda+\mu}+1\right)\left(\partial_{t} \xi_{p}^{n}, \xi_{p}^{n}\right) \geq \frac{1}{2 \Delta t}\left(\frac{1}{\lambda+\mu}+1\right)\left(\left\|\xi_{p}^{n}\right\|^{2}+\left\|\xi_{p}^{n-1}\right\|^{2}\right),
$$

and by using the relation (3.8), (3.9), we finally have the following relation

$$
\begin{aligned}
& \left(\frac{1}{\lambda+\mu}+1\right)\left(\partial_{t} \xi_{p}^{n}, \xi_{p}^{n}\right)+\left(K \nabla \xi_{p}^{n}, \nabla \xi_{p}^{n}\right)-\left(\mathcal{A} \xi_{\tilde{\sigma}}^{n}, \partial_{t} \xi_{\tilde{\sigma}}^{n}\right) \\
\geq & \frac{2(\lambda+\mu-1)-\epsilon}{4 \Delta t(\lambda+\mu)}\left\|\xi_{p}^{n}\right\|^{2}-\frac{\epsilon(\lambda+\mu+1)+1}{2 \epsilon \Delta t(\lambda+\mu)}\left\|\xi_{p}^{n-1}\right\|^{2}+\left\|K^{\frac{1}{2}} \nabla \xi_{p}^{n}\right\|^{2} .
\end{aligned}
$$

For the right hand side, we have the following estimation

$$
\begin{aligned}
& \left(\operatorname{tr}\left(\partial_{t} \tilde{\sigma}^{n}-\tilde{\sigma}_{t}^{n}\right), \xi_{p}^{n}\right) \leq 2\left\|\partial_{t} \tilde{\sigma}-\tilde{\sigma}_{t}^{n}\right\|^{2}+\epsilon_{1}\left\|\xi_{p}^{n}\right\|^{2} \\
& \leq C\left\|\frac{1}{\Delta t} \int_{t_{n-1}}^{t_{n}}\left(t_{n-1}-t\right) \tilde{\sigma}_{t t} d t\right\|^{2}+\epsilon_{1}\left\|\xi_{p}^{n}\right\|^{2} \leq C(\Delta t)^{2}+\epsilon_{1}\left\|\xi_{p}^{n}\right\|^{2}, \\
& \left(\operatorname{tr}\left(\partial_{t}\left(\tilde{\sigma}^{n}-R_{h}^{\tilde{\sigma}} \tilde{\sigma}^{n}\right)\right), \xi_{p}^{n}\right) \leq C\left\|\partial_{t}\left(\tilde{\sigma}^{n}-R_{h}^{\tilde{\sigma}} \tilde{\sigma}^{n}\right)\right\|^{2}+\epsilon_{2}\left\|\xi_{p}^{n}\right\|^{2} \\
& \leq C h^{2\left(k_{1}+1\right)}\left\|\tilde{\sigma}_{t}\right\|_{k_{1}+1}^{2}+\epsilon_{2}\left\|\xi_{p}^{n}\right\|^{2} .
\end{aligned}
$$


Similarly, we can also bound term related to $p$ as follows

$$
\begin{aligned}
& \left|\left(p_{t}^{n}-\partial_{t} p^{n}, \xi_{p}^{n}\right)\right| \leq C(\Delta t)^{2}+\epsilon_{3}\left\|\xi_{p}^{n}\right\|^{2}, \\
& \left|\left(\partial_{t}\left(p^{n}-R_{h}^{p} p^{n}\right), \xi_{p}^{n}\right)\right| \leq C h^{2\left(k_{2}+1\right)}\left\|p_{t}\right\|_{k_{2}+1}^{2}+\epsilon_{4}\left\|\xi_{p}^{n}\right\|^{2} .
\end{aligned}
$$

From all the estimation on the left and right hand side, we multiply $\Delta t$ on both side of the inequality, choose $\epsilon_{1}+\epsilon_{2}+\epsilon_{3}+\epsilon_{4}<\epsilon$ and sum from $n=1$ to $m$. Notice $\xi_{p}^{0}=0$, we finally get the following estimation

$$
\left\|\xi_{p}^{m}\right\|^{2}+C \sum_{k=1}^{m} \Delta t\left\|K^{1 / 2} \nabla p^{k}\right\| \lesssim(\Delta t)^{2}+h^{2\left(k_{1}+1\right)}+h^{2\left(k_{2}+1\right)} .
$$

Together with the property of projection $R_{h}^{p}$, we finally get the results in the theorem.

Similar as the convergence analysis of semidiscrete case, we can also prove the convergence of $\left\|\tilde{\sigma}^{n}-\tilde{\sigma}_{h}^{n}\right\|$ and $\left\|\boldsymbol{u}^{n}-\boldsymbol{u}_{h}^{n}\right\|$.

\section{Numerical experiments}

In this section, we will implement the finite element method proposed in Section 2 for the Biot consolidation problem. We test two examples with homogeneous Dirichlet boundary conditions and mixed boundary conditions respectively. We will consider the following system:

$$
\begin{array}{ll}
\frac{1}{2 \mu}\left[\tilde{\boldsymbol{\sigma}}-\frac{\lambda}{2(\lambda+\mu)} \operatorname{tr}(\tilde{\boldsymbol{\sigma}}) \cdot I\right]-\epsilon(\boldsymbol{u})+\frac{1}{2(\lambda+\mu)} p \cdot \boldsymbol{I}=0 & \text { in }\{t>0\} \times \Omega, \\
\operatorname{div} \tilde{\boldsymbol{\sigma}}=f & \text { in }\{t>0\} \times \Omega, \\
\frac{1}{2(\lambda+\mu)}(\operatorname{tr}(\tilde{\boldsymbol{\sigma}}))_{t}+\left(\frac{1}{\lambda+\mu}+1\right) p_{t}-\operatorname{div}(K \nabla p)=S_{f} & \text { in }\{t>0\} \times \Omega,
\end{array}
$$

with $\Omega=[0,1]^{2}$ and physical parameters in the implement are chosen as: $\lambda=1, K=1$, $\mu=0.5$. The initial condition are chosen as $p^{0}=0, \boldsymbol{u}^{0}=\mathbf{0}$.

In order to see the convergence order in the physical space, we fixed time step size $\Delta t$ such that errors in physical space dominate. In the numerical experiments, we mainly show the convergence order of $\tilde{\boldsymbol{\sigma}}^{n}, \boldsymbol{u}^{n}, p^{n}$ in relative error to different mesh sizes and different degrees of polynomial order at $n \Delta t$ (we choose the data of $n=3$ in the tables).

Example 4.1. In the first example, we verify the convergence order of finite element method for the poroelasticity problem with homogenous Dirichlet boundary conditions, 
Table 1: $k_{1}=3, k_{2}=2, \Delta t=0.001$.

\begin{tabular}{|c|c|c|c|c|c|c|c|c|}
\hline \multirow{2}{*}{$\frac{1}{h}$} & \multicolumn{2}{|c|}{$\frac{\left\|\tilde{\boldsymbol{\sigma}}-\tilde{\boldsymbol{\sigma}}_{h}\right\|_{0}}{\|\tilde{\boldsymbol{\sigma}}\|_{0}}$} & \multicolumn{2}{|c|}{$\frac{\left\|\operatorname{div}\left(\tilde{\boldsymbol{\sigma}}-\tilde{\sigma}_{h}\right)\right\|_{0}}{\|\operatorname{div} \tilde{\sigma}\|_{0}}$} & \multicolumn{2}{|c|}{$\frac{\left\|\boldsymbol{u}-\boldsymbol{u}_{h}\right\|_{0}}{\|\boldsymbol{u}\|_{0}}$} & \multicolumn{2}{|c|}{$\frac{\mid p-p_{h} \|_{1}}{\|p\|_{1}}$} \\
\hline & error & order & error & order & error & order & error & order \\
\hline 2 & $2.2229 \mathrm{e}-2$ & - & $2.7304 \mathrm{e}-2$ & - & $3.4461 \mathrm{e}-2$ & - & $2.0699 \mathrm{e}-2$ & - \\
\hline 4 & $2.8644 \mathrm{e}-3$ & 2.95 & $3.6303 \mathrm{e}-3$ & 2.91 & $4.2007 \mathrm{e}-3$ & 3.04 & $5.7468 \mathrm{e}-2$ & 1.85 \\
\hline 8 & $3.4950 \mathrm{e}-4$ & 3.04 & $4.6085 \mathrm{e}-4$ & 2.98 & $4.9245 \mathrm{e}-4$ & 3.09 & $1.4908 \mathrm{e}-3$ & 1.95 \\
\hline
\end{tabular}

Table 2: $k_{1}=3, k_{2}=3, \Delta t=0.001$.

\begin{tabular}{|c|c|c|c|c|c|c|c|c|}
\hline \multirow[b]{2}{*}{$\frac{1}{h}$} & \multicolumn{2}{|c|}{$\frac{\left\|\tilde{\boldsymbol{\sigma}}-\tilde{\boldsymbol{\sigma}}_{h}\right\|_{0}}{\|\tilde{\boldsymbol{\sigma}}\|_{0}}$} & \multicolumn{2}{|c|}{$\frac{\left\|\operatorname{div}\left(\tilde{\boldsymbol{\sigma}}-\tilde{\boldsymbol{\sigma}}_{h}\right)\right\|_{0}}{\|\operatorname{div} \tilde{\sigma}\|_{0}}$} & \multicolumn{2}{|c|}{$\frac{\left\|\boldsymbol{u}-\boldsymbol{u}_{h}\right\|_{0}}{\|\boldsymbol{u}\|_{0}}$} & \multicolumn{2}{|c|}{$\left\|p-p_{h}\right\|_{1}$} \\
\hline & $\frac{\| \tilde{\boldsymbol{\sigma}}}{\text { error }}$ & order & $\begin{array}{l}\| \text { div } \\
\text { error }\end{array}$ & order & error & order & error & order \\
\hline 2 & $1.5977 \mathrm{e}-2$ & - & $2.7304 \mathrm{e}-2$ & - & $2.9628 \mathrm{e}-2$ & - & $4.6950 \mathrm{e}-2$ & - \\
\hline 4 & 7.9767e-3 & 3.79 & $3.6303 \mathrm{e}-3$ & 2.91 & $3.7283 \mathrm{e}-3$ & 2.94 & $6.1560 \mathrm{e}-3$ & 2.93 \\
\hline 8 & $5.0558 \mathrm{e}-5$ & 3.98 & $4.6085 e-4$ & 2.98 & $4.76160 \mathrm{e}-4$ & 2.98 & $8.4300 \mathrm{e}-4$ & 2.87 \\
\hline
\end{tabular}

i.e., the exact solutions $\boldsymbol{u}, p$ satisfies $p=0, \boldsymbol{u}=\mathbf{0}$ on $\partial \Omega$. Explicit data are chosen as follows:

$$
\begin{aligned}
& \boldsymbol{u}=\left(\begin{array}{l}
u_{1} \\
u_{2}
\end{array}\right)=\left(\begin{array}{c}
t x(1-x) y(1-y) \\
t x(1-x) y(1-y)
\end{array}\right), \quad p=t \sin (\pi x) \sin (\pi y), \\
& \boldsymbol{\sigma}=\left(\begin{array}{cc}
(\lambda+2 \mu) \frac{\partial u_{1}}{\partial x}+\lambda \frac{\partial u_{2}}{\partial y} & \mu\left(\frac{\partial u_{1}}{\partial y}+\frac{\partial u_{2}}{\partial x}\right) \\
\mu\left(\frac{\partial u_{1}}{\partial y}+\frac{\partial u_{2}}{\partial x}\right) & (\lambda+2 \mu) \frac{\partial u_{2}}{\partial y}+\lambda \frac{\partial u_{1}}{\partial x}
\end{array}\right), \quad \tilde{\sigma}=\left(\begin{array}{cc}
\sigma_{11}-c_{u p} p & \sigma_{12} \\
\sigma_{12} & \sigma_{22}-c_{u p} p
\end{array}\right), \\
& f=-\nabla \cdot \tilde{\sigma}=\left(\begin{array}{c}
f_{1} \\
f_{2}
\end{array}\right)=\left(\begin{array}{cc}
\frac{\partial \tilde{\sigma}_{11}}{\partial x}+\frac{\partial \tilde{\sigma}_{12}}{\partial y} \\
\frac{\partial \tilde{\sigma}_{12}}{\partial x}+\frac{\partial \tilde{\sigma}_{22}}{\partial y}
\end{array}\right), \quad S_{f}=\frac{\partial}{\partial t}\left(c_{u p} \nabla \cdot \boldsymbol{u}\right)+\frac{\partial}{\partial t}\left(c_{p p} p\right)-\nabla \cdot(K \nabla p) .
\end{aligned}
$$

In the numerical experiments, we utilize two different kinds of finite elements. We first use $p_{3}$ (polynomials with degree less or equal to 3 ) finite elements for variable $\tilde{\sigma}$ and piecewise discontinuous quadratic element for displacement $u$. For pressure $p$, we use the piecewise continuous quadratic finite element which corresponding to $k_{1}=3, k_{2}=2$ in the main result of Theorem 3.2. From the numerical results in Table 1, we can see pressure variable $p$ has optimal convergence order in both $L^{2}$ and energy norm and $\tilde{\sigma}, \boldsymbol{u}$ has one order lower, which is consistent with the theoretical results in Theorem 3.2 since the convergence order of $\tilde{\sigma}$ is constrained by polynomial degree $k_{2}$ of pressure variable $p$. We also test the example with $k_{1}=3$ for $\tilde{\sigma}$ and $k_{2}=3$ for the pressure $p$ and numerical results are shown in Table 2 in which the both $\tilde{\sigma}, u, p$ have the optimal convergence order and the numerical implementation is consistent with the theoretical results.

Example 4.2. In this second experiment, we will test the finite element method for the poroelasticity problem with mixed boundary condition. We choose the function $f(t, x)$, 
Table 3: Example 4.2: $k_{1}=3, k_{2}=2, \Delta t=10^{-6}$.

\begin{tabular}{|c|c|c|c|c|c|c|c|c|}
\hline \multirow{2}{*}{$\frac{1}{h}$} & \multicolumn{2}{|c|}{$\frac{\left\|\tilde{\boldsymbol{\sigma}}-\tilde{\boldsymbol{\sigma}}_{h}\right\|_{0}}{\|\tilde{\boldsymbol{\sigma}}\|_{0}}$} & \multicolumn{2}{|c|}{$\frac{\left\|\operatorname{div}\left(\tilde{\sigma}-\tilde{\sigma}_{h}\right)\right\|_{0}}{\|\operatorname{div} \tilde{\sigma}\|_{0}}$} & \multicolumn{2}{|c|}{$\frac{\left\|\boldsymbol{u}-\boldsymbol{u}_{h}\right\|_{0}}{\|\boldsymbol{u}\|_{0}}$} & \multicolumn{2}{|c|}{$\frac{\left\|p-p_{h}\right\|_{1}}{\|p\|_{1}}$} \\
\hline & error & order & error & order & error & order & error & order \\
\hline 2 & 0.0265 & - & 0.0917 & - & 0.0527 & - & 0.2198 & - \\
\hline 4 & 0.0031 & 3.09 & 0.0128 & 2.84 & 0.0067 & 2.97 & 0.0598 & 1.88 \\
\hline 8 & $4.1743 \mathrm{e}-4$ & 2.90 & 0.0017 & 2.95 & $8.5900 \mathrm{e}-4$ & 2.97 & 0.0153 & 1.97 \\
\hline
\end{tabular}

Table 4: Example 4.2: $k_{1}=3, k_{2}=3, \Delta t=10^{-6}$.

\begin{tabular}{|c|c|c|c|c|c|c|c|c|}
\hline \multirow{2}{*}{$\frac{1}{h}$} & \multicolumn{2}{|c|}{$\frac{\left\|\tilde{\boldsymbol{\sigma}}-\tilde{\boldsymbol{\sigma}}_{h}\right\|_{0}}{\|\tilde{\boldsymbol{\sigma}}\|_{0}}$} & \multicolumn{2}{|c|}{$\frac{\left\|\operatorname{div}\left(\tilde{\boldsymbol{\sigma}}-\tilde{\boldsymbol{\sigma}}_{h}\right)\right\|_{0}}{\|\operatorname{div} \tilde{\boldsymbol{\sigma}}\|_{0}}$} & \multicolumn{2}{|c|}{$\frac{\left\|\boldsymbol{u}-\boldsymbol{u}_{h}\right\|_{0}}{\|\boldsymbol{u}\|_{0}}$} & \multicolumn{2}{|c|}{$\frac{\left\|p-p_{h}\right\|_{1}}{\|p\|_{1}}$} \\
\hline & error & order & error & order & error & order & error & order \\
\hline 2 & 0.0023 & - & 0.0917 & - & 0.0509 & - & 0.0599 & - \\
\hline 4 & 0.0017 & 3.77 & 0.0728 & 2.84 & 0.0067 & 2.93 & 0.0082 & 2.88 \\
\hline 8 & $1.0640 \mathrm{e}-4$ & 3.98 & 0.0017 & 2.95 & $8.5700 \mathrm{e}-4$ & 2.96 & 0.0011 & 2.95 \\
\hline
\end{tabular}

$S_{f}(t, x)$ such that the exact solutions of the model problem are

$$
\begin{aligned}
& \boldsymbol{u}=\left(\begin{array}{l}
u_{1} \\
u_{2}
\end{array}\right)=\left(\begin{array}{c}
t \sin \pi x \sin ^{2} \pi y \\
t \sin \pi x \sin \pi y
\end{array}\right), \quad p=\pi(\lambda+2 \mu) t \sin \pi x \cos \pi y, \\
& \tilde{\boldsymbol{\sigma}}=\left(\begin{array}{cc}
(\lambda+2 \mu) \pi t \cos \pi x \sin ^{2} \pi y-2 \mu \pi t \sin \pi x \cos \pi y & \mu \pi t(\sin \pi x \sin 2 \pi y+\cos \pi x \sin \pi y) \\
\mu \pi t(\sin \pi x \sin 2 \pi y+\cos \pi x \sin \pi y) & \lambda \pi t \cos \pi x \sin ^{2} \pi y
\end{array}\right),
\end{aligned}
$$

which satisfy the following mixed boundary conditions

$$
\begin{array}{llll}
p=0 & \text { on }\{x=0, x=1\}, & k \nabla p \cdot \boldsymbol{n}=0 & \text { on }\{y=0, y=1\}, \\
\boldsymbol{u}=0 & \text { on }\{x=0, x=1\}, & \tilde{\boldsymbol{\sigma}} \cdot \boldsymbol{n}=0 & \text { on }\{y=0, y=1\} .
\end{array}
$$

Then, the closed form of $f, S_{f}$ is

$$
\begin{aligned}
& f=\nabla \cdot \tilde{\sigma}=\left(\begin{array}{c}
-(\lambda+2 \mu) \pi^{2} t \sin \pi x \sin ^{2} \pi y \\
(\lambda+2 \mu) \pi^{2} t \cos \pi x \sin 2 \pi y-\mu \pi^{2} t \sin \pi x \sin \pi y
\end{array}\right), \\
& S_{f}=\frac{\partial}{\partial t}\left(c_{u p} \nabla \cdot u\right)+\frac{\partial}{\partial t}\left(c_{p p} p\right)-\nabla \cdot(K \nabla p) .
\end{aligned}
$$

We use finite elements with $k_{1}=3, k_{2}=2$ as well as $k_{1}=k_{2}=3$ for this mixed boundary model problem. We fixed $\Delta t=10^{-6}$ and choose $h=1 / 2,1 / 4,1 / 8$ to see the convergence order of $h$. From Tables 3 and 4 , we can see that all the variables $\tilde{\sigma}^{n}, \boldsymbol{u}^{n}, p^{n}$ have optimal convergence order with respect to mesh size $h$.

\section{Conclusions}

In this paper, we mainly study using the finite element methods for the poroelasticity problem. Mixed finite element method with three variables: total stress tensor, displace- 
ment and pore pressure of the model problem are studied. Conforming finite element method with strong symmetric element for the stress tensor and piecewise discontinuous finite element for the displacement [11] are used for the linear elasticity subproblem. For the pore pressure of the fluid, we use the piecewise continuous Lagrange finite element method. Theoretical analysis shows that the finite element method has optimal convergence order. Numerical experiments are implemented to verify the theoretical results. In our future work, we will consider the fast solver for the discrete linear algebraic system based on this finite element methods.

\section{Acknowledgements}

L. P. Chen is supported by the National Natural Science Foundation of China under Grant Nos. 11501473, 11426189 and the Fundamental Research Funds for the Central Universities of China (No. 2682016CX108).

\section{References}

[1] Douglas N. ARnold, Richard S. Falk And Ragnar Winther, Finite element exterior calculus, homological techniques, and applications, Acta Numer., 15 (2006), pp. 1-155.

[2] Douglas N. Arnold, Richard S. Falk And Ragnar Winther, Finite element exterior calculus: from Hodge theory to numerical stability, Bulletin of the American Mathematical Society, 47(2) (2010), pp. 281-354.

[3] Roza Asadi, Behzad Ataieashtiani and Craig T. Simmons, Finite volume coupling strategies for the solution of a Biot consolidation model, Comput. Geotech., 55 (2014), pp. 494505.

[4] Trygve Baerland, Jeonghun J Lee, Kent Andre Mardal and Ragnar Winther, Weakly imposed symmetry and robust preconditioners for Biot's consolidation model, Comput. Methods Appl. Math., 17 (2017).

[5] Maurice A. Biot, General theory of three-demensional conslidation, J. Appl. Phys., 12(2) (1941), pp. 155-164.

[6] Maurice A. Biot, General solutions of the equations of elasticity and Consolidation for porous material, J. Appl. Mech., (1958).

[7] K. Brenan, S. CAmpbell AND L. Petzold, Numerical Solution of Initial-Value Problems in Differential-Algebraic Equations, volume 14, Society for Industrial and Applied Mathematics (SIAM), Philadelphia, 1996.

[8] F. J. Gaspar, F. J. Lisbona, C. W. OOsterleE, AND R. Wienands, A systematic comparison of coupled and distributive smoothing in multigrid for the poroelasticity system, Numer. Linear Algebra Appl., 11(23) (2004), pp. 93-113.

[9] F. J. GASPAR, F. J. LisBOnA AND P. N. VABISHCHEVICH, A finite difference analysis of Biot's consolidation model, Appl. Numer. Math., 44(4) (2003), pp. 487-506.

[10] QingGuO HONG AND JOHANNES KRAUS, Parameter-robust stability of classical three-field formulation of BIOT's consolidation model, Electronic Trans. Numer. Anal., 49 (2018), pp. 210-243.

[11] JUN Hu AND SHANGYOU ZHANG, A family of conforming mixed finite elements for linear elasticity on triangular grids, Mathematics, 58(2) (2014), pp. 297-307. 
[12] J. KorsaWe AND G. STARKE, A least-square mixed finite element method for Biot's consolidation problem in porous media, SIAM J. Numer. Anal., 43(1) (2005), pp. 318-339.

[13] A. F. MAK, Lidu HuANG AND QINQUe WANG, A biphasic poroelastic analysis of the flow dependent subcutaneous tissue pressure and compaction due to epidermal loadings: issues in pressure sore, J. Biomech. Eng., 116(4) (1994), pp. 421-429.

[14] A. M. MARcio, T. VidAR AND A. F. D. Loula, Asymptotic behavior of semidiscrete finiteelement approximations of Biot' s consolidation problem, SIAM J. Numer. Anal., 33(3) (1996), pp. 1065-1083.

[15] Joseph P. Morris, Yue HaO, William Foxall and Walt McNab, A study of injectioninduced mechanical deformation at the in salah $\mathrm{Co}_{2}$ storage project, Int. J. Greenhouse Gas Control, 5(2) (2011), pp. 270-280.

[16] Joseph P. Morris, Walt W. McNab, Susan K Carroll, Yue HaO, William Foxall AND JEFFREY L WAGONER, Injection and reservoir hazard management: The role of injectioninduced mechanical deformation and geochemical alteration at in salah $\mathrm{CO}_{2}$ storage project: Status reportquarter end, June 2009. Technical Report, LLNL Technical Report, 2009.

[17] V. C. MoW, M. K. KWAN, W. M. LAI AND M. H. Holmes, A finite deformation theory for nonlinearly permeable soft hydrated biological tissues, Frontiers in Biomechanics, pages 153-179, Springer, 1986.

[18] M. A. Murad AND A. F. D. LoulA, Improved accuracy in finite element analysis of Biot's consolidation problem, Comput. Methods Appl. Mech. Eng., 95(3) (1992), pp. 359-382.

[19] JAN MARTIN NORDBOtTEN, Stable cell-centered finite volume discretization for Biot equations, SIAM J. Numer. Anal., 54(2) (2016), pp. 942-968.

[20] Phillip Joseph Phillips And Mary F. Wheeler, A coupling of mixed and continuous Galerkin finite element methods for poroelasticity I: the continuous in time case, Comput. Geosci., 11(2) (2007), pp. 131-144.

[21] Phillip Joseph Phillips And Mary F. Wheeler, A coupling of mixed and continuous Galerkin finite element methods for poroelasticity II: the discrete-in-time case, Comput. Geosci., 11(2) (2007), pp. 145-158.

[22] Phillip Joseph Phillips And Mary F. Wheeler, A coupling of mixed and discontinuous Galerkin finite-element methods for poroelasticity, Comput. Geosci., 12(4) (2008), pp. 417-435.

[23] C. Rodrigo, F. J. GASPAR, X. HU AND L. T. ZIKATANOV, Stability and monotonicity for some discretizations of the Biot's consolidation model, Comput. Methods Appl. Mech. Eng., 298 (2016), pp. 183-204.

[24] C. Rodrigo, X. Hu, P. Ohm, J. H. Adler, F. J. Gaspar And L. T. Zikatanov, New stabilized discretizations for poroelasticity and the Stokes' equations, Comput. Methods Appl. Mech. Eng., 341 (2018), pp. 467-484.

[25] B. R. Simon, M. V. Kaufmann, M. A. McAfee And A. L. Baldwin, Finite element models for arterial wall mechanics, J. Biomech. Eng., 115(4B) (1993), pp. 489-496.

[26] Maria Tchonkova, John Peters And Stein StUre, A new mixed finite element method for poro-elasticity, Int. J. Numer. Anal. Methods Geomech., 32 (2008), pp.579-606.

[27] KARL VON TERZAGHI, Erdbaumechanik auf bodenphysikalischer Grundlage, Deuticke, 1925.

[28] Ming YANG AND LARRY A. TABer, The possible role of poroelasticity in the apparent viscoelastic behavior of passive cardiac muscle, J. Biomech., 24(7) (1991), pp. 587-597.

[29] SON-YOUNG YI, Convergence analysis of a new mixed finite element method for Biot's consolidation model, Numer. Methods Partial Differential Equasion, 23(11) (2007), pp. 904-922.

[30] SON YOUNG YI, A coupling of nonconforming and mixed finite element methods for Biot's consoli- 
dation model, Numer. Methods Partial Differential Equations, 29(5) (2013), pp. 1749-1777.

[31] SON Young YI, Convergence analysis of a new mixed finite element method for Biot's consolidation model, Numer. Methods Partial Differential Equations, 30(4) (2014), pp. 1189-1210. 